\title{
Quantization of the particle with a linear massless solution
}

\author{
Renann Lipinski Jusinskas \\ Institute of Physics of the Czech Academy of Sciences, \\ CEICO - Central European Institute for Cosmology and Fundamental Physics, \\ Na Slovance 2, 182 21, Prague, Czech Republic \\ E-mail: renannlj@fzu.cz
}

ABSTRACT: In this work, a solution linear in the momentum for the massless constraint $P^{m} P_{m}=0$ is investigated. It is presented in terms of a $\mathrm{SO}(2 n, \mathbb{C})$ to $\mathrm{U}(n)$ decomposition and interpreted in terms of projective pure spinors, which are known to parametrize the $\frac{\mathrm{SO}(2 n)}{\mathrm{U}(n)}$ coset. The worldline action is quantized using the BRST formalism and, using the results of Berkovits and Cherkis, the ghost number zero wave function is shown to generate massless solutions for field equations of arbitrary spin. The model can be covariantly expressed by the action recently proposed in $D=10$ by Berkovits, in terms of a twistorlike constraint. However, a thorough account of its gauge symmetries does not lead to a spacetime supersymmetric theory. In order to derive from first principles the superparticle in the pure spinor formalism, a new model is proposed with partial worldline supersymmetry. The gauge algebra is then analyzed within the Batalin-Vilkovisky formalism and the gauge fixed action is finally shown to describe the pure spinor superparticle times a $\mathrm{U}(1)$ decoupled sector.

KeYwords: BRST Quantization, Gauge Symmetry, Superstrings and Heterotic Strings

ARXIV EPRINT: 1808.07463 


\section{Contents}

1 Overview 1

2 The linear massless solution $\quad 4$

2.1 Quantization and cohomology 4

2.2 Projective pure spinors and the twistor-like constraint 6

$\begin{array}{ll}2.3 \text { Covariant formulation in } D=10 & 7\end{array}$

3 The pure spinor superparticle $\quad 8$

$\begin{array}{ll}3.1 \text { Master action } & 9\end{array}$

$\begin{array}{ll}3.2 & \text { Gauge fixing } \\ 3.3\end{array}$

$\begin{array}{lll}3.3 & \text { Ghost number twisting and cohomology } & 15\end{array}$

4 Summary and final remarks $\quad 16$

\section{Overview}

The worldline action for a massless particle in Minkowski space (metric $\eta^{m n}$ ) is given by

$$
S_{p}=\int d \tau\left\{P_{m} \dot{X}^{m}-\frac{1}{2} e \eta^{m n} P_{m} P_{n}\right\}
$$

where $X^{m}(\tau)$ denotes the coordinates of the particle at a given instant $\tau, \dot{X}^{m} \equiv \frac{d X^{m}}{d \tau}, P_{m}$ stands for the conjugate momentum of $X^{m}$ and the einbein $e$ is the Lagrange multiplier imposing the mass-shell. By construction, $S_{p}$ has a gauge symmetry characterized by the invariance under reparametrization of $\tau$.

Quantization of the action (1.1) leads to a scalar field $\varphi$ satisfying the wave equation. Within the BRST formalism, $\varphi$ appears as the ghost number zero cohomology of the BRST charge

$$
Q=c P^{m} P_{m},
$$

where $(b, c)$ is the ghost conjugate pair associated to reparametrization symmetry and the gauge fixed action takes the form

$$
S=\int d \tau\left\{P_{m} \dot{X}^{m}-\frac{1}{2} P_{m} P^{m}+b \dot{c}\right\}
$$

Note that the choice $e=0$ is not physically sensible as it corresponds to a degenerate worldline metric.

Understanding the massless particle is particularly interesting because it can be viewed in some sense as the zero length limit of the string. Therefore, alternative formulations of 
the particle action might provide some insight to develop different string descriptions. The quantization of the particle has been extensively explored in the past (see [1] and references therein for a review). Among the different techniques, the twistor parametrization introduced by Penrose in $D=4$ [2] is one of the most fruitful. In [3], for example, the twistor description was extended from massless to massive particles, including AdS backgrounds.

In this work, the quantization of the massless particle will be investigated with a particular solution for the mass-shell condition. In order to do so, the Lorentz group $\mathrm{SO}(2 n-1,1)$ will be described in terms of a complexified $\mathrm{SO}(2 n)$ group and the worldline fields will be presented in terms of $\mathrm{U}(n)$ representations. For $X^{m}$ and $P_{m}$ the decomposition is

$$
\begin{aligned}
X^{m} & =\left\{x^{a}, \bar{x}_{a}\right\}, \\
P_{m} & =\left\{p_{a}, \bar{p}^{a}\right\},
\end{aligned}
$$

with $m=1, \ldots, 2 n$ and $a=1, \ldots, n$, such that

$$
\begin{aligned}
x^{a} \equiv \frac{1}{\sqrt{2}}\left(X^{2 a-1}+i X^{2 a}\right), & p_{a} \equiv \frac{1}{\sqrt{2}}\left(P_{2 a-1}-i P_{2 a}\right), \\
\bar{x}_{a} \equiv \frac{1}{\sqrt{2}}\left(X^{2 a-1}-i X^{2 a}\right), & \bar{p}^{a} \equiv \frac{1}{\sqrt{2}}\left(P_{2 a-1}+i P_{2 a}\right),
\end{aligned}
$$

and the massless condition $P^{m} P_{m}=0$ is rewritten as

$$
\bar{p}^{a} p_{a}=0
$$

Any solution of (1.6) in $D=2 n$ dimensions can be put in the form

$$
\bar{p}^{a}+\gamma^{a b} p_{b}=0
$$

for some antisymmetric $\mathrm{U}(n)$ tensor $\gamma^{a b}$. As will be shown here, (1.7) is the irreducible part of the twistor-like constraint

$$
\left(\gamma^{m} \lambda\right)_{\alpha} P_{m}=0
$$

proposed in [4], where $\gamma^{m}$ denotes a chiral block of the Dirac matrices and $\lambda^{\alpha}$ is a projective pure spinor, with $\alpha=1, \ldots, 2^{n-1}$. Therefore, $\gamma^{a b}$ can be interpreted in terms of projective pure spinors, known to parametrize the $\frac{\mathrm{SO}(2 n)}{\mathrm{U}(n)}$ coset [5].

In $[6,7]$, Hughston showed that solutions to the wave equation in even dimensions can be constructed using projective pure spinors. Later on, this idea was extended with the proper definition of the projective pure spinors integration measure and the explicit construction of solutions of massless field equations of arbitrary spin [8].

As it turns out, the BRST quantization of the massless particle action subjected to the constraint (1.7) gives rise to a similar structure and its ghost number zero cohomology has a simple form which can be expressed by the wave function

$$
F=F\left(\mathbb{X}^{a}, \gamma^{a b}\right)
$$

Here, $\mathbb{X}^{a}=x^{a}+\gamma^{a b} \bar{x}_{b}$ corresponds to the independent components of the spinor $\mathbb{X}_{\alpha}$ defined by

$$
\mathbb{X}_{\alpha} \equiv X^{m}\left(\gamma_{m} \lambda\right)_{\alpha}
$$


In [8], it was shown that $F$ is the generating function of massless solutions of field equations of spin $\frac{N}{2}$, given by

$$
\phi^{\left(\alpha_{1} \cdots \alpha_{N}\right)}=\int[d \lambda] \lambda^{\alpha_{1}} \ldots \lambda^{\alpha_{N}} F\left(\mathbb{X}_{\alpha}, \lambda^{\alpha}\right)
$$

where $[d \lambda]$ is some integration measure over the projective pure spinors.

More recently [9], the constraint (1.8) was implemented in an effort to provide a bona fide gauge structure that would finally lead to the pure spinor superparticle [10]. However, the wave function (1.9) encodes a field content far richer than the physical spectrum of the superparticle. In addition, a more extensive analysis of the gauge symmetries of the worldline action proposed in [9] does not lead to a spacetime supersymmetric theory.

On the other hand, the analysis of the physical degrees of freedom of the tendimensional model promptly hints at its generalization. The new ingredients are the anticommuting spinor $\bar{\theta}^{\alpha}$, a "superpartner" for the pure spinor $\lambda^{\alpha}$, satisfying

$$
\left(\lambda \gamma^{m} \bar{\theta}\right)=0
$$

with conjugate $\bar{p}_{\alpha}$, and a sort of local worldline supersymmetry for the spacetime spinors, generated by $\lambda^{\alpha} \bar{p}_{\alpha}$. The gauge fixed action of this model and its associated BRST charge can be cast as

$$
\begin{aligned}
S_{P S} & =\int d \tau\left\{P_{m} \dot{X}^{m}+w_{\alpha} \dot{\lambda}^{\alpha}-\frac{1}{2} P_{m} P^{m}+\bar{p}_{\alpha} \dot{\bar{\theta}}^{\alpha}+\hat{p}_{\alpha} \dot{\hat{\theta}}^{\alpha}+\bar{\Omega} \dot{\Omega}+\beta \dot{\gamma}\right\}, \\
Q & =\gamma \lambda^{\alpha} \bar{p}_{\alpha}-\left(\lambda \gamma^{m} \hat{\theta}\right) P_{m}+\Omega\left(\beta \gamma-\hat{\theta}^{\alpha} \hat{p}_{\alpha}-\lambda^{\alpha} w_{\alpha}\right) .
\end{aligned}
$$

Here, the conjugate pairs $\left\{\hat{p}_{\alpha}, \hat{\theta}^{\alpha}\right\},\{\bar{\Omega}, \Omega\}$ and $\{\beta, \gamma\}$ respectively denote the ghosts for the twistor-like symmetry, scaling symmetry and worldline supersymmetry.

Because of the reducibility of $(1.8), \bar{p}_{\alpha}$ is constrained to satisfy

$$
\left(\lambda \gamma^{m} \gamma^{n} \bar{p}\right)=0
$$

However, by introducing the unconstrained spinors

$$
\begin{aligned}
& \theta^{\alpha} \equiv \bar{\theta}^{\alpha}+\gamma^{-1} \hat{\theta}^{\alpha}, \\
& p_{\alpha} \equiv \bar{p}_{\alpha}+\gamma \hat{p}_{\alpha},
\end{aligned}
$$

spacetime supersymmetry becomes manifest, similarly to what was done in [11], giving rise to the pure spinor superparticle times a U(1) decoupled sector. The partial worldline supersymmetry can then be viewed as a tool to implement spacetime supersymmetry.

This work is organized as follows. In section 2, the BRST quantization of the worldline action with constraint (1.7) is analyzed and the ghost number zero cohomology is expressed as the wave function (1.9). The constraints (1.7) and (1.8) are shown to be equivalent and the model is covariantly quantized. In section 3, using the Batalin-Vilkovisky formalism, the extension of Berkovits' proposal in [9] is analyzed. The master action is built taking into account the pure spinor constraint and derived symmetries, which appear as constraints for the antifields. The gauge fixing procedure leading to the action (1.13a) is carried out and, after a simple set of field redefinitions, the pure spinor superparticle action is obtained. Section 4 presents a quick summary and some final remarks. 


\section{The linear massless solution}

After the field decomposition introduced in (1.5), the action (1.1) can be simply rewritten as

$$
S_{p}=\int_{C} d \tau\left\{p_{a} \dot{x}^{a}+\bar{p}^{a} \dot{\bar{x}}_{a}-e \bar{p}^{a} p_{a}\right\}
$$

Now, instead of the massless constraint $\bar{p}^{a} p_{a}=0$, consider a solution linear in the momentum of the form

$$
\bar{p}^{a}+\gamma^{a b} p_{b}=0
$$

where $\gamma^{a b}$ is an antisymmetric tensor. The issue with this solution is it automatically breaks the $\mathrm{U}(n)$ symmetry. To amend this, $\gamma^{a b}$ is promoted to a dynamical field with conjugate $\beta_{a b}$, such that the worldline action for this system is given by

$$
S=\int_{C} d \tau\left\{p_{a} \dot{x}^{a}+\bar{p}^{a} \dot{\bar{x}}_{a}+\frac{1}{2} \beta_{a b} \dot{\gamma}^{a b}-L_{a}\left(\bar{p}^{a}+\gamma^{a b} p_{b}\right)\right\}
$$

where $L_{a}$ is the Lagrange multiplier of the constraint (2.2). Observe that even if the term $e \bar{p}^{a} p_{a}$ is kept in the action, a field redefinition of the form

$$
L_{a} \rightarrow L_{a}-e p_{a},
$$

can absorb the einbein e.

\subsection{Quantization and cohomology}

The action (2.3) is invariant under the local transformations parametrized by $\left\{c, c_{a}, d\right\}$,

$$
\begin{aligned}
\delta x^{a} & =c \dot{x}^{a}-\gamma^{a b} c_{b}+d \bar{p}^{a}, \\
\delta \bar{x}_{a} & =c \dot{\bar{x}}_{a}+c_{a}+d p_{a}, \\
\delta p_{a} & =c \dot{p}_{a}, \\
\delta \bar{p}^{a} & =c \dot{\bar{p}}^{a}, \\
\delta \gamma^{a b} & =c \dot{\gamma}^{a b}, \\
\delta \beta_{a b} & =c \dot{\beta}_{a b}+\left(p_{a} c_{b}-p_{b} c_{a}\right), \\
\delta L_{a} & =\frac{d}{d \tau}\left(c L_{a}\right)+\dot{c}_{a}+\dot{d} p_{a},
\end{aligned}
$$

which represent respectively reparametrization symmetry and the symmetries generated by $(2.2)$ and $\bar{p}^{a} p_{a}$. However, these gauge symmetries are not all independent, $c f$. the field shift (2.4), and the above transformations are invariant up to equations of motion by

$$
\begin{aligned}
\delta^{\prime} c & =\phi_{0}, \\
\delta^{\prime} c_{a} & =-\phi_{0} L_{a}-\phi_{1} P_{a}, \\
\delta^{\prime} d & =\phi_{1},
\end{aligned}
$$

with $\phi_{0}$ and $\phi_{1}$ parametrizing the redundant gauge symmetries. 
Because of the gauge-for-gauge transformations (2.6), the gauge symmetries parametrized by $c$ and $d$ may be disregarded. It is straightforward then to apply the BRST construction and obtain the gauge fixed action with its associated BRST charge. Naively, the gauge $L_{a}=0$ could be chosen, but it is easy to see that worldline reparametrization would reappear as a residual gauge symmetry. This is related to the field shift (2.4), for such gauge choice would imply the degeneracy of the worldline metric. Conveniently choosing $L_{a}=p_{a}$, the gauge fixed action is

$$
S=\int d \tau\left\{p_{a} \dot{x}^{a}+\bar{p}^{a} \dot{\bar{x}}_{a}+\frac{1}{2} \beta_{a b} \dot{\gamma}^{a b}-\bar{p}^{a} p_{a}-b^{a} \dot{c}_{a}\right\}
$$

with BRST charge

$$
Q=c_{a}\left(\bar{p}^{a}+\gamma^{a b} p_{b}\right)
$$

Here, $c_{a}$ is the gauge parameter promoted to an anticommuting ghost number +1 field and $b^{a}$ is its canonical conjugate with ghost number -1 .

The canonical (anti)commutation relations obtained from (2.7) are given by

$$
\begin{aligned}
{\left[x^{a}, p_{b}\right] } & =i \delta_{b}^{a}, \\
{\left[\bar{x}_{a}, \bar{p}^{b}\right] } & =i \delta_{a}^{b}, \\
{\left[\gamma^{a b}, \beta_{c d}\right] } & =i\left(\delta_{c}^{a} \delta_{d}^{b}-\delta_{d}^{a} \delta_{c}^{b}\right), \\
\left\{c_{a}, b^{b}\right\} & =i \delta_{a}^{b},
\end{aligned}
$$

and can be used to determine the BRST transformations of the worldline fields,

$$
\begin{aligned}
\delta x^{a} & =c_{b} \gamma^{b a}, & \delta \gamma^{a b} & =0, \\
\delta \bar{x}_{a} & =c_{a}, & \delta \beta_{a b} & =p_{a} c_{b}-p_{b} c_{a}, \\
\delta p_{a} & =0, & \delta c_{a} & =0, \\
\delta \bar{p}^{a} & =0, & \delta b^{a} & =\bar{p}^{a}+\gamma^{a b} p_{b} .
\end{aligned}
$$

To evaluate the cohomology of $Q$, consider a generic wave function built from $x^{a}, \bar{x}_{a}$, $\gamma^{a b}$ and $c_{a}$,

$$
\Psi(c, x, \bar{x}, \gamma)=F(x, \bar{x}, \gamma)+c_{a} F^{a}(x, \bar{x}, \gamma)+c_{a} c_{b} F^{a b}(x, \bar{x}, \gamma)+\ldots
$$

where the expansion in the $c_{a}$ 's is finite because of its anticommuting character, i.e. $(c)^{n+1}=$ 0 . Within the BRST construction, physical states should be annihilated by $Q$, i.e. $Q \Psi=0$. It is straightforward to compute the action of the BRST charge and at ghost number zero it implies the equation of motion

$$
\bar{\partial}^{a} F+\gamma^{a b} \partial_{b} F=0
$$

where $\partial_{a} \equiv \frac{\partial}{\partial x^{a}}$ and $\bar{\partial}^{a} \equiv \frac{\partial}{\partial \bar{x}_{a}}$. Other than $\bar{\partial}^{a} \partial_{a} F=0$, the above equation does not clearly provide any dynamical information. Observe however that by defining a new field, $\mathbb{X}^{a}$, as

$$
\mathbb{X}^{a} \equiv x^{a}+\gamma^{a b} \bar{x}_{b}
$$


then any function $F$ of the form

$$
F=F(\mathbb{X}, \gamma)
$$

satisfies the equation of motion (2.12). Such solutions were explored in [8] in generic even dimensions, where $\gamma^{a b}$ was interpreted as a projective pure spinor. Using the techniques introduced by Berkovits and Cherkis it is possible to demonstrate that the wave function $F(\mathbb{X}, \gamma)$ generates massless solutions for field equations of arbitrary integer and half-integer spin. The spin of a given solution is an extra input and appears in the integration over the projective pure spinors.

\subsection{Projective pure spinors and the twistor-like constraint}

The connection between $\gamma^{a b}$ and projective pure spinors remains to be explained. The constraint $(2.2)$ can be generically expressed in $D=2 n$ dimensions as a chiral spinor constraint of the form

$$
\left(\gamma^{m} \lambda\right)_{\alpha} P_{m}=0
$$

where $\gamma^{m}$ denotes a chiral block of the Dirac matrices and $\lambda^{\alpha}$ is a projective pure spinor. The twistor-like constraint (2.15) was proposed by Berkovits in $D=10$ [4] as an attempt to explain the origin of the pure spinor formalism [12].

At the level of the action, (2.15) is implemented through a Lagrange multiplier $L^{\alpha}$ and it is straightforward to show that it is equivalent to (2.2):

$D=4 \quad$ For $n=2$, antichiral and chiral spinors are represented by dotted and undotted indices. In terms of $\mathrm{U}(2)$ indices, $L_{\dot{\alpha}}$ is expressed as a vector $L_{a}$, with $a=1,2$, while a chiral spinor is expressed as $\lambda_{\alpha}=\left(\lambda_{+}, \lambda_{-}\right)$. Being a projective pure spinor, $\lambda_{\alpha}$ can be parametrized as $\lambda_{+}=1$ and $\lambda_{-}=\gamma$. Therefore,

$$
\left(L \gamma^{m} \lambda\right) P_{m}=L_{a}\left(\bar{p}^{a}+\gamma \epsilon^{a b} p_{b}\right) .
$$

Note that in this case the antisymmetric tensor $\gamma^{a b}$ has only one independent component, expressed here as $\gamma^{a b} \equiv \gamma \epsilon^{a b}$, with $\epsilon^{12}=-\epsilon^{21}=1$.

$D=6 \quad$ For $n=3$, chiral and antichiral spinors have the same indices. The Lagrange multiplier is expressed as $L_{\alpha}=\left(L_{+}, L_{a}\right)$, with $a=1,2,3$, and the projective pure spinor as $\lambda_{\alpha}=\left(1, \gamma_{a}\right)$. Therefore,

$$
\left(L \gamma^{m} \lambda\right) P_{m}=\left(L_{a}+\gamma_{a} L_{+}\right)\left(\bar{p}^{a}-\epsilon^{a b c} p_{b} \gamma_{c}\right) .
$$

The antisymmetric tensor $\gamma^{a b}$ can expressed as a vector $\gamma_{a} \equiv \frac{1}{2} \epsilon_{a b c} \gamma^{a b}$, where $\epsilon^{a b c}$ and $\epsilon_{a b c}$ are a totally antisymmetric tensors with $\epsilon^{123}=\epsilon_{321}=1$.

$D=8 \quad$ For $n=4$, antichiral and chiral spinors are represented by dotted and undotted indices. In terms of $\mathrm{U}(4)$ indices, $L^{\dot{\alpha}}$ can be split into one fundamental and one antifundamental representation, $L_{a}$ and $L^{a}$, while a projective pure spinor can be parametrized as $\lambda^{\alpha}=\left(1, \gamma^{a b}, \frac{1}{8} \epsilon_{a b c d} \gamma^{a b} \gamma^{c d}\right)$, where $\epsilon_{a b c d}$ is the totally antisymmetric $\mathrm{U}(4)$ tensor with $\epsilon_{1234}=1$. Using this parametrization, $\left(L \gamma^{m} \lambda\right) P_{m}$ can be expressed as:

$$
\left(L \gamma^{m} \lambda\right) P_{m}=\left(L_{a}+\frac{1}{2} \epsilon_{a c d e} \gamma^{d e} L^{c}\right)\left(\bar{p}^{a}+\gamma^{a b} p_{b}\right) .
$$


$D=10 \quad$ For $n=5$, chiral and antichiral spinors have the same indices. The Lagrange multiplier can be expressed as $L^{\alpha}=\left(L^{+}, L^{a b}, L_{a}\right)$, with $a=1, \ldots, 5$, and the projective pure spinor as $\lambda^{\alpha}=\left(1, \gamma^{a b},-\frac{1}{8} \epsilon_{a b c d e} \gamma^{b c} \gamma^{d e}\right)$, in which $\epsilon_{a b c d e}$ is the totally antisymmetric $\mathrm{U}(5)$ tensor with $\epsilon_{12345}=1$. Then, it is possible to show that:

$$
\left(L \gamma^{m} \lambda\right) P_{m}=\left(L_{a}-\frac{1}{8} \epsilon_{a b c d e} \gamma^{b c} \gamma^{d e} L^{+}+\frac{1}{4} \epsilon_{a b c d e} \gamma^{d e} L^{b c}\right)\left(\bar{p}^{a}+\gamma^{a b} p_{b}\right) .
$$

Notice that in every case $L_{a}$ constitutes the only independent Lagrange multiplier, confirming that (2.2) is the irreducible part of the twistor-like constraint (2.15).

\subsection{Covariant formulation in $D=10$}

In spite of its form, (2.15) is not Lorentz covariant because of the projective condition on the pure spinor. A natural way of solving this problem is to use instead a pure spinor endowed with an extra local scaling symmetry. This solution was brought forth in [9] through the ten-dimensional action

$$
S_{B}=\int d \tau\left\{P_{m} \dot{X}^{m}+w_{\alpha} \dot{\lambda}^{\alpha}+A w_{\alpha} \lambda^{\alpha}-\left(L \gamma^{m} \lambda\right) P_{m}\right\}
$$

with $\alpha=1, \ldots, 16$, where $\lambda^{\alpha}$ is a pure spinor satisfying

$$
\left(\lambda \gamma^{m} \lambda\right)=0
$$

$w_{\alpha}$ is its conjugate. The Lagrange multipliers $\left\{L^{\alpha}, A\right\}$ are associated respectively to the twistor-like symmetry and to a scaling symmetry acting as

$$
\begin{aligned}
\delta \lambda^{\alpha} & =\Omega \lambda^{\alpha}, \quad \delta w_{\alpha}=-\Omega w_{\alpha}, \\
\delta L^{\alpha} & =-\Omega L^{\alpha}, \quad \delta A=-\dot{\Omega},
\end{aligned}
$$

with parameter $\Omega$. Note that the scaling preserves the constraint $(2.20)$ and $\lambda^{\alpha}$ can then be fundamentally described as a projective pure spinor. Furthermore, the action (2.19) has an intrinsic gauge symmetry due to the pure spinor constraint and it is invariant under the transformation $\delta w_{\alpha}=\epsilon_{m}\left(\gamma^{m} \lambda\right)_{\alpha}$, with local parameter $\epsilon_{m}$.

In [9], using an argument based on BRST symmetry, the reducibility of the twistor-like constraint was partially disregarded (leading to unconstrained ghosts) and the quantization of (2.19) was proposed to give rise to the pure spinor superparticle [10]. However, this cannot be the case, as evidenced by the previous cohomology analysis (subsection 2.1). In fact, a proper account of the gauge symmetries of the action (2.19) uncovers a chain of symmetries connected to the pure spinor condition (2.20) which, in the context of the Batalin-Vilkovisky formalism, effectively appear as constraints on the antifields. This will be further explored in section 3 with the construction of the master action in a similar setup. For now, it is enough to know that a covariant gauge can be chosen, leading to the gauge fixed action

$$
S=\int d \tau\left\{P_{m} \dot{X}^{m}-\frac{1}{2} P_{m} P^{m}+w_{\alpha} \dot{\lambda}^{\alpha}+\hat{p}_{\alpha} \dot{\hat{\theta}}^{\alpha}+\bar{\Omega} \dot{\Omega}\right\},
$$


where $\left\{\hat{\theta}^{\alpha}, \hat{p}_{\alpha}\right\}$ is the constrained ghost conjugate pair for the twistor-like symmetry and $\{\Omega, \bar{\Omega}\}$ is the ghost conjugate pair for the scaling symmetry. The BRST charge is given by

$$
Q=\left(\lambda \gamma^{m} \hat{\theta}\right) P_{m}+\Omega\left(\lambda^{\alpha} w_{\alpha}+\hat{\theta}^{\alpha} \hat{p}_{\alpha}\right)
$$

The absence of spacetime supersymmetry in the action (2.22) can be explained by the extra constraint connecting $\hat{p}_{\alpha}$ to $\lambda^{\alpha}$, given by

$$
\left(\lambda \gamma^{m} \gamma^{n} \hat{p}\right)=0
$$

which arises from the reducibility of (2.15), cf. (2.18), and implies yet another intrinsic gauge symmetry for the gauge fixed action (2.22),

$$
\begin{aligned}
\delta w_{\alpha} & =\epsilon_{m n}\left(\hat{p} \gamma^{m n}\right)_{\alpha}+\epsilon \hat{p}_{\alpha}, \\
\delta \hat{\theta}^{\alpha} & =\epsilon_{m n}\left(\gamma^{m n} \lambda\right)^{\alpha}+\epsilon \lambda^{\alpha},
\end{aligned}
$$

where $\epsilon$ and $\epsilon_{m n}$ are local parameters and $\gamma^{m n}=\frac{1}{2}\left[\gamma^{m}, \gamma^{n}\right]$. After taking into account these symmetries, it is straightforward to show that the BRST charge (2.23) has the same physical spectrum of the $D=10$ version of (2.8).

\section{The pure spinor superparticle}

In this section a new proposal is presented to extend Berkovits' twistor-like constraint with (partial) worldline supersymmetry, finally leading to the pure spinor superparticle.

Consider an extension of (2.19) with the inclusion of an anticommuting variable $\bar{\theta}^{\alpha}$ satisfying

$$
\left(\lambda \gamma^{m} \bar{\theta}\right)=0
$$

together with a sort of worldline supersymmetry for the spacetime spinors. Such a model can be expressed by the action

$$
S_{0}=\int d \tau\left\{P_{m} \dot{X}^{m}+w_{\alpha} \nabla \lambda^{\alpha}-L^{\alpha}\left(\gamma^{m} \lambda\right)_{\alpha} P_{m}+\bar{p}_{\alpha} \dot{\bar{\theta}}^{\alpha}+\chi \lambda^{\alpha} \bar{p}_{\alpha}\right\},
$$

where $\bar{p}_{\alpha}$ is the conjugate of $\bar{\theta}^{\alpha}, \chi$ is the Lagrange multiplier of the local symmetry generator $\lambda^{\alpha} \bar{p}_{\alpha}$ and $\nabla$ is the covariant derivative for the scaling symmetry with gauge field $A$.

Due to the constraints (2.20) and (3.1), the action is invariant under the transformations

$$
\begin{aligned}
\delta w_{\alpha} & =d_{m}\left(\gamma^{m} \lambda\right)_{\alpha}+e_{m}\left(\gamma^{m} \bar{\theta}\right)_{\alpha}, \\
\delta \bar{p}_{\alpha} & =e_{m}\left(\gamma^{m} \lambda\right)_{\alpha}, \\
\delta L^{\alpha} & =f \lambda^{\alpha}+f_{m n}\left(\gamma^{m n} \lambda\right)^{\alpha}+g \bar{\theta}^{\alpha},
\end{aligned}
$$

where $d_{m}, e_{m}, f, f_{m n}$ and $g$ are local parameters. From now on, these will be called pure spinor symmetries. Having a special role, they are not to be gauge fixed and instead appear as constraints on the antifields in the construction of the master action. 
Keeping this in mind, the gauge symmetries of (3.2) are summarized by

$$
\begin{aligned}
\delta X^{m} & =c \dot{X}^{m}+\left(\lambda \gamma^{m} \hat{\theta}\right) \\
\delta P_{m} & =c \dot{P}_{m} \\
\delta \lambda^{\alpha} & =c \dot{\lambda}^{\alpha}+\Omega \lambda^{\alpha} \\
\delta w_{\alpha} & =c \dot{w}_{\alpha}-P_{m}\left(\gamma^{m} \hat{\theta}\right)_{\alpha}-\Omega w_{\alpha}+\gamma \bar{p}_{\alpha}, \\
\delta L^{\alpha} & =\frac{d}{d \tau}\left(c L^{\alpha}\right)+\nabla \hat{\theta}^{\alpha}-\Omega L^{\alpha} \\
\delta A & =\frac{d}{d \tau}(c A)-\dot{\Omega} \\
\delta \bar{\theta}^{\alpha} & =c \dot{\bar{\theta}}^{\alpha}+\gamma \lambda^{\alpha} \\
\delta \bar{p}_{\alpha} & =c \dot{\bar{p}}_{\alpha} \\
\delta \chi & =\frac{d}{d \tau}(c \chi)+\nabla \gamma-\Omega \chi
\end{aligned}
$$

where the parameters $c, \Omega, \hat{\theta}^{\alpha}$ and $\gamma$ respectively denote worldline reparametrization, scaling symmetry, twistor-like symmetry and the worldline supersymmetry of the spacetime spinors. These gauge symmetries, however, are not irreducible. Consider the following transformations of the gauge parameters,

$$
\begin{aligned}
\delta^{\prime} c & =\phi, \\
\delta^{\prime} \Omega & =\phi A, \\
\delta^{\prime} \hat{\theta}^{\alpha} & =-\phi L^{\alpha}, \\
\delta^{\prime} \gamma & =-\phi \chi .
\end{aligned}
$$

Here, $\phi$ is the gauge-for-gauge parameter. It is straightforward to show that they leave the gauge transformations invariant up to equations of motion,

$$
\begin{aligned}
\delta^{\prime}\left[\delta X^{m}\right] & =\phi\left\{\dot{X}^{m}-\left(\lambda \gamma^{m} L\right)\right\}, & \delta^{\prime}[\delta A] & =0, \\
\delta^{\prime}\left[\delta P_{m}\right] & =\phi\left\{\dot{P}_{m}\right\}, & \delta^{\prime}\left[\delta \bar{\theta}^{\alpha}\right] & =\phi\left\{\nabla \bar{\theta}^{\alpha}-\chi \lambda^{\alpha}\right\} \\
\delta^{\prime}\left[\delta \lambda^{\alpha}\right] & =\phi\left\{\nabla \lambda^{\alpha}\right\}, & \delta^{\prime}\left[\delta \bar{p}_{\alpha}\right] & =\phi\left\{\nabla \bar{p}_{\alpha}\right\}, \\
\delta^{\prime}\left[\delta w_{\alpha}\right] & =\phi\left\{\nabla w_{\alpha}-\chi \bar{p}_{\alpha}+P_{m}\left(\gamma^{m} L\right)_{\alpha}\right\}, & \delta^{\prime}[\delta \chi] & =0 .
\end{aligned}
$$

\subsection{Master action}

The Batalin-Vilkovisky (BV) formalism seems to be the most suitable tool to deal with the gauge structure of the action (3.2). In order to build the master action, the field content of the model is extended by promoting gauge and gauge-for-gauge parameters to fields. They will be collectively denoted by $\Phi^{I}$, where $I$ is an index running over all fields:

$$
\Phi^{I} \equiv\left\{X^{m}, P_{m}, \lambda^{\alpha}, w_{\alpha}, L^{\alpha}, A, \bar{\theta}^{\alpha}, \bar{p}_{\alpha}, \chi, c, \Omega, \hat{\theta}^{\alpha}, \gamma, \phi\right\} .
$$

Note that $\left\{c, \Omega, \hat{\theta}^{\alpha}\right\}$ are Grassmann odd while $\{\gamma, \phi\}$ are Grassmann even fields. The antifields, denoted by $\Phi_{I}^{*}$, have the opposite statistics of the fields and will be defined as

$$
\Phi_{I}^{*} \equiv\left\{X_{m}^{*}, P_{*}^{m}, \lambda_{\alpha}^{*}, w_{*}^{\alpha}, L_{\alpha}^{*}, A^{*}, \bar{\theta}_{\alpha}^{*}, \bar{p}_{*}^{\alpha}, \chi^{*}, c^{*}, \Omega^{*}, \hat{\theta}_{\alpha}^{*}, \gamma^{*}, \phi^{*}\right\} .
$$


Fields and antifields are used to define the antibrackets between any operator pair $\mathcal{O}_{1}$ and $\mathcal{O}_{2}$

$$
\left\{\mathcal{O}_{1}, \mathcal{O}_{2}\right\} \equiv \mathcal{O}_{1}\left(\frac{\overleftarrow{\partial}}{\partial \Phi_{I}^{*}} \frac{\partial}{\partial \Phi^{I}}-\frac{\overleftarrow{\partial}}{\partial \Phi^{I}} \frac{\partial}{\partial \Phi_{I}^{*}}\right) \mathcal{O}_{2}
$$

where the sum over $I$ is implicit. In particular,

$$
\left\{\Phi_{I}^{*}, \Phi^{J}\right\}=\delta_{I}^{J} .
$$

Due to the pure spinor symmetries of (3.3), the antifields are constrained to satisfy

$$
\begin{aligned}
\left(\lambda \gamma^{m} w_{*}\right) & =0, \\
\left(\lambda \gamma^{m} \bar{p}_{*}\right) & =0, \\
\bar{\theta}^{\alpha} L_{\alpha}^{*} & =0, \\
\left(\lambda \gamma^{m} \gamma^{n} L^{*}\right) & =0 .
\end{aligned}
$$

Observe that treating (3.3) as ordinary gauge transformations leads to an infinite tower of gauge-for-gauge symmetries. Instead, the problem is simplified if the above conditions are imposed. There is an apparent contradiction between the constraints (2.20), (3.1) and (3.11), and the field-antifield conjugation operation (3.10). For example,

$$
\begin{aligned}
\left\{\lambda_{\alpha}^{*},\left(\lambda \gamma^{m} \lambda\right)\right\} & =2\left(\gamma^{m} \lambda\right)_{\alpha}, \\
& \neq 0
\end{aligned}
$$

but the pure spinor (gauge) symmetries implied by the constraints have to be taken into account. This is a known feature of the pure spinor beta-gamma system and it can be easily solved by using only gauge invariant operators. The pure spinor symmetries will be further discussed later.

The master action can be cast as

$$
S=S_{0}+S_{1}+S_{2}+S_{3},
$$

where $S_{0}$ is displayed in (3.2) and

$$
\begin{aligned}
S_{1}= & \int d \tau\left\{c \dot{X}^{m} X_{m}^{*}+c \dot{P}_{m} P_{*}^{m}+c \dot{\lambda}^{\alpha} \lambda_{\alpha}^{*}+c \dot{w}_{\alpha} w_{*}^{\alpha}-c L^{\alpha} \dot{L}_{\alpha}^{*}-c A \dot{A}^{*}\right\} \\
& +\int d \tau\left\{c \dot{\bar{p}}_{\alpha} \bar{p}_{*}^{\alpha}+c \dot{\bar{\theta}}^{\alpha} \bar{\theta}_{\alpha}^{*}-c \chi \dot{\chi}^{*}+\gamma \lambda^{\alpha} \bar{\theta}_{\alpha}^{*}+\gamma \bar{p}_{\alpha} w_{*}^{\alpha}+(\nabla \gamma) \chi^{*}\right\} \\
& +\int d \tau\left\{\left(\lambda \gamma^{m} \hat{\theta}\right) X_{m}^{*}-P_{m}\left(\hat{\theta} \gamma^{m} w_{*}\right)+\nabla \hat{\theta}^{\alpha} L_{\alpha}^{*}\right\} \\
& +\int d \tau\left\{\Omega \lambda^{\alpha} \lambda_{\alpha}^{*}-\Omega w_{\alpha} w_{*}^{\alpha}-\Omega L^{\alpha} L_{\alpha}^{*}-\Omega \chi \chi^{*}-\dot{\Omega} A^{*}\right\}, \\
S_{2}= & \int d \tau\left\{c \dot{c} c^{*}+c \dot{\Omega} \Omega^{*}+c \dot{\gamma} \gamma^{*}+c \dot{\hat{\theta}}^{\alpha} \hat{\theta}_{\alpha}^{*}-\Omega \hat{\theta}^{\alpha} \hat{\theta}_{\alpha}^{*}-\Omega \gamma \gamma^{*}\right\} \\
& +\int d \tau\left\{\phi c^{*}+\phi A \Omega^{*}-\phi L^{\alpha} \hat{\theta}_{\alpha}^{*}-\phi \chi \gamma^{*}+c \dot{\phi} \phi^{*}-\dot{c} \phi \phi^{*}\right\}, \\
S_{3}= & \int d \tau\left\{\phi P_{*}^{m} X_{m}^{*}+\phi w_{*}^{\alpha} \lambda_{\alpha}^{*}-\phi \bar{\theta}_{\alpha}^{*} \bar{p}_{*}^{\alpha}\right\} .
\end{aligned}
$$


$S_{1}$ accounts for the gauge transformations (3.4), $S_{2}$ is associated to the extension of the gauge algebra to the ghost fields and the gauge-for-gauge symmetries (3.5), and $S_{3}$ (quadratic in the antifields) is necessary because of the on-shell invariance of the gauge transformations, cf. (3.6).

The master action (3.13) satisfies the master equation,

$$
\{S, S\}=0,
$$

and, for any operator $\mathcal{O}$, it defines its BV transformation, given by

$$
\delta_{\mathrm{BV}} \mathcal{O} \equiv\{S, \mathcal{O}\}
$$

Satisfying the master equation is equivalent to the statement that the master action is invariant under the BV transformations. For completeness, the transformations of the fields are

$$
\begin{aligned}
\delta_{\mathrm{BV}} X^{m} & =c \dot{X}^{m}+\left(\lambda \gamma^{m} \hat{\theta}\right)+\phi P_{*}^{m}, \\
\delta_{\mathrm{BV}} P_{m} & =c \dot{P}_{m}-\phi X_{m}^{*}, \\
\delta_{\mathrm{BV}} \lambda^{\alpha} & =c \dot{\lambda}^{\alpha}+\Omega \lambda^{\alpha}+\phi w_{*}^{\alpha}, \\
\delta_{\mathrm{BV}} w_{\alpha} & =c \dot{w}_{\alpha}-P_{m}\left(\hat{\theta} \gamma^{m}\right)_{\alpha}-\Omega w_{\alpha}+\gamma \bar{p}_{\alpha}-\phi \lambda_{\alpha}^{*}, \\
\delta_{\mathrm{BV}} L^{\alpha} & =\frac{d}{d \tau}\left(c L^{\alpha}\right)+\nabla \hat{\theta}^{\alpha}-\Omega L^{\alpha}, \\
\delta_{\mathrm{BV}} A & =\frac{d}{d \tau}(c A)-\dot{\Omega}, \\
\delta_{\mathrm{BV}} \bar{\theta}^{\alpha} & =c \overline{\bar{\theta}}^{\alpha}+\gamma \lambda^{\alpha}-\phi \bar{p}_{*}^{\alpha}, \\
\delta_{\mathrm{BV}} \bar{p}_{\alpha} & =c \dot{\bar{p}}_{\alpha}-\phi \bar{\theta}_{\alpha}^{*}, \\
\delta_{\mathrm{BV}} \chi & =\frac{d}{d \tau}(c \chi)+\nabla \gamma-\Omega \chi, \\
\delta_{\mathrm{BV}} c & =c \dot{c}+\phi, \\
\delta_{\mathrm{BV}} \Omega & =c \dot{\Omega}+\phi A, \\
\delta_{\mathrm{BV}} \hat{\theta}^{\alpha} & =c \dot{\hat{\theta}}-\Omega \hat{\theta}^{\alpha}-\phi L^{\alpha}, \\
\delta_{\mathrm{BV}} \gamma & =c \dot{\gamma}-\Omega \gamma-\phi \chi, \\
\delta_{\mathrm{BV}} \phi & =c \dot{\phi}-\dot{c} \phi,
\end{aligned}
$$

and the transformation of the antifields are

$$
\begin{aligned}
\delta_{\mathrm{BV}} X_{m}^{*} & =\dot{P}_{m}+\frac{d}{d \tau}\left(c X_{m}^{*}\right), \\
\delta_{\mathrm{BV}} P_{*}^{m} & =-\dot{X}^{m}+\left(L \gamma^{m} \lambda\right)+\frac{d}{d \tau}\left(c P_{*}^{m}\right)+\left(\hat{\theta} \gamma^{m} w_{*}\right), \\
\delta_{\mathrm{BV}} \lambda_{\alpha}^{*} & =\nabla w_{\alpha}+\left(\gamma^{m} L\right)_{\alpha} P_{m}-\chi \bar{p}_{\alpha}+\frac{d}{d \tau}\left(c \lambda_{\alpha}^{*}\right)-\left(\gamma^{m} \hat{\theta}\right)_{\alpha} X_{m}^{*}-\gamma \bar{\theta}_{\alpha}^{*}-\Omega \lambda_{\alpha}^{*}, \\
\delta_{\mathrm{BV}} w_{*}^{\alpha} & =-\nabla \lambda^{\alpha}+\frac{d}{d \tau}\left(c w_{*}^{\alpha}\right)+\Omega w_{*}^{\alpha}, \\
\delta_{\mathrm{BV}} L_{\alpha}^{*} & =\left(\gamma^{m} \lambda\right)_{\alpha} P_{m}+c \dot{L}_{\alpha}^{*}+\Omega L_{\alpha}^{*}+\phi \hat{\theta}_{\alpha}^{*},
\end{aligned}
$$




$$
\begin{aligned}
\delta_{\mathrm{BV}} A^{*}= & c \dot{A}^{*}-w_{\alpha} \lambda^{\alpha}+\hat{\theta}^{\alpha} L_{\alpha}^{*}+\gamma \chi^{*}-\phi \Omega^{*}, \\
\delta_{\mathrm{BV}} \bar{\theta}_{\alpha}^{*}= & \dot{\bar{p}}_{\alpha}+\frac{d}{d \tau}\left(c \bar{\theta}_{\alpha}^{*}\right), \\
\delta_{\mathrm{BV}} \bar{p}_{*}^{\alpha}= & \dot{\bar{\theta}}^{\alpha}-\chi \lambda^{\alpha}+\frac{d}{d \tau}\left(c \bar{p}_{*}^{\alpha}\right)+\gamma w_{*}^{\alpha}, \\
\delta_{\mathrm{BV}} \chi^{*}= & \lambda^{\alpha} \bar{p}_{\alpha}+c \dot{\chi}^{*}+\Omega \chi^{*}-\phi \gamma^{*}, \\
\delta_{\mathrm{BV}} c^{*}= & \dot{X}^{m} X_{m}^{*}+\dot{P}_{m} P_{m}^{*}+\dot{\lambda}^{\alpha} \lambda_{\alpha}^{*}+\dot{w}_{\alpha} w_{*}^{\alpha}-L^{\alpha} \dot{L}_{\alpha}^{*}-A \dot{A}^{*}+\dot{\bar{p}}_{\alpha} \bar{p}_{*}^{\alpha}+\dot{\bar{\theta}}^{\alpha} \bar{\theta}_{\alpha}^{*} \\
& -\chi \dot{\chi}^{*}+2 \dot{c} c^{*}+c \dot{c}^{*}+\dot{\gamma} \gamma^{*}+\dot{\hat{\theta}}^{\alpha} \hat{\theta}_{\alpha}^{*}+\dot{\Omega} \Omega^{*}+2 \dot{\phi} \phi^{*}+\phi \dot{\phi}^{*}, \\
\delta_{\mathrm{BV}} \Omega^{*}= & \lambda^{\alpha} \lambda_{\alpha}^{*}-w_{\alpha} w_{*}^{\alpha}-L^{\alpha} L_{\alpha}^{*}+\dot{A}^{*}-\chi \chi^{*}+\frac{d}{d \tau}\left(c \Omega^{*}\right)-\hat{\theta}^{\alpha} \hat{\theta}_{\alpha}^{*}-\gamma \gamma^{*}, \\
\delta_{\mathrm{BV}} \hat{\theta}_{\alpha}^{*}= & \left(\lambda \gamma^{m}\right)_{\alpha} X_{m}^{*}-P_{m}\left(\gamma^{m} w_{*}\right)_{\alpha}-\nabla L_{\alpha}^{*}+\frac{d}{d \tau}\left(c \hat{\theta}_{\alpha}^{*}\right)+\Omega \hat{\theta}_{\alpha}^{*}, \\
\delta_{\mathrm{BV}} \gamma^{*}= & \nabla \chi^{*}-\lambda^{\alpha} \bar{\theta}_{\alpha}^{*}-\bar{p}_{\alpha} w_{*}^{\alpha}+\frac{d}{d \tau}\left(c \gamma^{*}\right)+\Omega \gamma^{*}, \\
\delta_{\mathrm{BV}} \phi^{*}= & 2 \dot{c} \phi^{*}+c \dot{\phi}^{*}-c^{*}-A \Omega^{*}+L^{\alpha} \hat{\theta}_{\alpha}^{*}+\chi \gamma^{*}+\bar{\theta}_{\alpha}^{*} \bar{p}_{*}^{\alpha}-P_{*}^{m} X_{m}^{*}-w_{*}^{\alpha} \lambda_{\alpha}^{*} .
\end{aligned}
$$

As mentioned before, the pure spinor conditions (2.20) and (3.1) imply a series of additional constraints on the antifields which can be summarized as

$$
\begin{aligned}
\left(\lambda \gamma^{m} w_{*}\right) & =0, \\
\left(\lambda \gamma^{m} \bar{p}_{*}\right)+\left(\bar{\theta} \gamma^{m} w_{*}\right) & =0, \\
\bar{\theta}^{\alpha} L_{\alpha}^{*} & =0, \\
\lambda^{\alpha} L_{\alpha}^{*} & =0, \\
\left(\lambda \gamma^{m n} L^{*}\right) & =0, \\
\bar{p}_{*}^{\alpha} L_{\alpha}^{*}+\bar{\theta}^{\alpha} \hat{\theta}_{\alpha}^{*} & =0, \\
\lambda^{\alpha} \hat{\theta}_{\alpha}^{*}+w_{*}^{\alpha} L_{\alpha}^{*} & =0, \\
\left(\lambda \gamma^{m n} \hat{\theta}^{*}\right)+\left(w_{*} \gamma^{m n} L^{*}\right) & =0 .
\end{aligned}
$$

It is easy to check their consistency with the BV transformations. Naturally, such constraints are associated to the pure spinor gauge transformations and the master action is invariant under the pure spinor symmetries

$$
\begin{aligned}
\delta w_{\alpha}= & d_{m}\left(\gamma^{m} \lambda\right)_{\alpha}+e_{m}\left(\gamma^{m} \bar{\theta}\right)_{\alpha}-\bar{f} L_{\alpha}^{*}+\bar{f}_{m n}\left(\gamma^{m n} L^{*}\right)_{\alpha}, \\
\delta \bar{p}_{\alpha}= & e_{m}\left(\gamma^{m} \lambda\right)_{\alpha}+\bar{g} L_{\alpha}^{*}, \\
\delta L^{\alpha}= & f \lambda^{\alpha}+f_{m n}\left(\gamma^{m n} \lambda\right)^{\alpha}+g \bar{\theta}^{\alpha}+\bar{f} w_{*}^{\alpha}+\bar{f}_{m n}\left(\gamma^{m n} w_{*}\right)^{\alpha}+\bar{g} \bar{p}_{*}^{\alpha}, \\
\delta \hat{\theta}^{\alpha}= & \bar{f} \lambda^{\alpha}+\bar{f}_{m n}\left(\gamma^{m n} \lambda\right)^{\alpha}+\bar{g} \bar{\theta}^{\alpha}, \\
\delta \lambda_{\alpha}^{*}= & b_{m}\left(\gamma^{m} \lambda\right)_{\alpha}+c_{m}\left(\gamma^{m} \bar{\theta}\right)_{\alpha}-d_{m}\left(\gamma^{m} w_{*}\right)_{\alpha}-e_{m}\left(\gamma^{m} \bar{p}_{*}\right)_{\alpha} \\
& -f L_{\alpha}^{*}+f_{m n}\left(\gamma^{m n} L^{*}\right)_{\alpha}-\bar{f} \hat{\theta}_{\alpha}^{*}+\bar{f}_{m n}\left(\gamma^{m n} \hat{\theta}^{*}\right)_{\alpha}, \\
\delta \bar{\theta}_{\alpha}^{*}= & c_{m}\left(\gamma^{m} \lambda\right)_{\alpha}+e_{m}\left(\gamma^{m} w_{*}\right)_{\alpha}+g L_{\alpha}^{*}-\bar{g} \hat{\theta}_{\alpha}^{*},
\end{aligned}
$$

where $b_{m}, c_{m}, d_{m}, e_{m}, f, f_{m n}, g, \bar{f}, \bar{f}_{m n}$ and $\bar{g}$ are local parameters. 


\subsection{Gauge fixing}

A systematic procedure to fix the gauge symmetries of the theory involves the introduction of the non-minimal master action through auxiliary variables organized as antighosts, $\bar{\Phi}_{i}$, Nakanishi-Lautrup fields, $\Lambda_{i}$, and their respective antifields, $\bar{\Phi}_{*}^{i}$ and $\Lambda_{*}^{i}$, where the index $i$ usually denotes the gauge and gauge-for-gauge symmetries to be fixed. The non-minimal action is defined as

$$
\tilde{S}=S+\int d \tau \bar{\Phi}_{*}^{i} \Lambda_{i}
$$

and, by construction, it satisfies the master equation in the extended phase space.

In the present case,

$$
\begin{aligned}
\bar{\Phi}_{i} & \equiv\left\{b, \bar{\Omega}, \hat{p}_{\alpha}, \beta, \bar{\phi}\right\}, \\
\Lambda_{i} & \equiv\left\{B, O, M_{\alpha}, G, F\right\}, \\
\bar{\Phi}_{*}^{i} & \equiv\left\{b^{*}, \bar{\Omega}^{*}, \hat{p}_{*}^{\alpha}, \beta^{*}, \bar{\phi}^{*}\right\}, \\
\Lambda_{*}^{i} & \equiv\left\{B^{*}, O^{*}, M_{*}^{\alpha}, G^{*}, F^{*}\right\},
\end{aligned}
$$

where $\left\{b, \bar{\Omega}, \hat{p}_{\alpha}, \beta, \bar{\phi}\right\}$ are respectively the antighosts of $\left\{c, \Omega, \hat{\theta}^{\alpha}, \gamma, \phi\right\}$, and so on.

The gauge fixing fermion will be chosen to be

$$
\Xi=\int d \tau\left\{\hat{p}_{\alpha}\left[P_{m} \frac{\left(\gamma^{m} C\right)^{\alpha}}{2(C \lambda)}-L^{\alpha}\right]+\bar{\phi} c+\beta \chi+\bar{\Omega} A\right\}
$$

imposing the gauges $A=c=\chi=0$ and

$$
L^{\alpha}=P_{m} \frac{\left(\gamma^{m} C\right)^{\alpha}}{2(C \lambda)}
$$

where $C_{\alpha}$ is a constant spinor. The particular choice for $L^{\alpha}$ is related to the discussion just before equation (2.7) and the degeneracy of the worldline metric, and the gauge-for-gauge symmetry is being used to eliminate the reparametrization symmetry. At the level of the non-minimal master action, the gauge fixing is implemented by evaluating $\tilde{S}$ at

$$
\begin{aligned}
\Phi_{I}^{*} & =\frac{\delta \Xi}{\delta \Phi^{I}}, \\
\bar{\Phi}_{*}^{i} & =\frac{\delta \Xi}{\delta \Phi_{i}}, \\
\Lambda_{*}^{i} & =\frac{\delta \Xi}{\delta \Lambda_{i}},
\end{aligned}
$$

and the non-vanishing antifields are given here by

$$
\begin{aligned}
P_{*}^{m} & =\frac{\left(\hat{p} \gamma^{m} C\right)}{2(C \lambda)}, \\
\lambda_{\alpha}^{*} & =-P_{m} \frac{\left(\hat{p} \gamma^{m} C\right)}{2(C \lambda)^{2}} C_{\alpha}, \\
L_{\alpha}^{*} & =-\hat{p}_{\alpha} \\
A^{*} & =\bar{\Omega},
\end{aligned}
$$




$$
\begin{aligned}
\chi^{*} & =\beta, \\
c^{*} & =\bar{\phi}, \\
\bar{\Omega}^{*} & =A, \\
\beta^{*} & =\chi, \\
\bar{\phi}^{*} & =c .
\end{aligned}
$$

Note that these solutions have to be consistent with the antifield constraints of (3.19), thus

$$
\begin{aligned}
\bar{\theta}^{\alpha} \hat{p}_{\alpha} & =0, \\
\lambda^{\alpha} \hat{p}_{\alpha} & =0, \\
\left(\lambda \gamma^{m n} \hat{p}\right) & =0 .
\end{aligned}
$$

The gauge-fixed action, after solving for the equations of motion of the NakanishiLautrup fields, is simply

$$
S_{P S}=\int d \tau\left\{P_{m} \dot{X}^{m}+w_{\alpha} \dot{\lambda}^{\alpha}-\frac{1}{2} P_{m} P^{m}+\bar{p}_{\alpha} \dot{\bar{\theta}}^{\alpha}+\hat{p}_{\alpha} \dot{\hat{\theta}}^{\alpha}+\bar{\Omega} \dot{\Omega}+\beta \dot{\gamma}\right\},
$$

and it is invariant under the pure spinor symmetries

$$
\begin{aligned}
\delta w_{\alpha} & =d_{m}\left(\gamma^{m} \lambda\right)_{\alpha}+e_{m}\left(\gamma^{m} \bar{\theta}\right)_{\alpha}+\bar{f} \hat{p}_{\alpha}-\bar{f}_{m n}\left(\gamma^{m n} \hat{p}\right)_{\alpha}, \\
\delta \bar{p}_{\alpha} & =e_{m}\left(\gamma^{m} \lambda\right)_{\alpha}-\bar{g} \hat{p}_{\alpha}, \\
\delta \hat{\theta}^{\alpha} & =\bar{f} \lambda^{\alpha}+\bar{f}_{m n}\left(\gamma^{m n} \lambda\right)^{\alpha}+\bar{g} \bar{\theta}^{\alpha} .
\end{aligned}
$$

The BV-BRST transformations can be easily read from (3.17) and (3.18) and are given by

$$
\begin{aligned}
\delta X^{m} & =\left(\lambda \gamma^{m} \hat{\theta}\right) \\
\delta P_{m} & =0 \\
\delta \lambda^{\alpha} & =\Omega \lambda^{\alpha} \\
\delta w_{\alpha} & =-P_{m}\left(\hat{\theta} \gamma^{m}\right)_{\alpha}-\Omega w_{\alpha}+\gamma \bar{p}_{\alpha}, \\
\delta \bar{\theta}^{\alpha} & =\gamma \lambda^{\alpha} \\
\delta \bar{p}_{\alpha} & =0 \\
\delta \Omega & =0 \\
\delta \bar{\Omega} & =-w_{\alpha} \lambda^{\alpha}+\beta \gamma+\hat{p}_{\alpha} \hat{\theta}^{\alpha} \\
\delta \hat{\theta}^{\alpha} & =-\Omega \hat{\theta}^{\alpha} \\
\delta \hat{p}_{\alpha} & =-\left(\gamma^{m} \lambda\right)_{\alpha} P_{m}+\Omega \hat{p}_{\alpha}, \\
\delta \gamma & =-\Omega \gamma \\
\delta \beta & =\lambda^{\alpha} \bar{p}_{\alpha}+\Omega \beta .
\end{aligned}
$$

Using Noether's theorem, the BRST charge is computed to be

$$
Q=\gamma \lambda^{\alpha} \bar{p}_{\alpha}-\left(\lambda \gamma^{m} \hat{\theta}\right) P_{m}+\Omega\left(\beta \gamma-\hat{\theta}^{\alpha} \hat{p}_{\alpha}-\lambda^{\alpha} w_{\alpha}\right) .
$$

Note that the terms involving the constant spinor $C_{\alpha}$ decouple from the action and from the transformations above, as they are proportional to the on-shell vanishing ghost-for-ghost $\phi$. 


\subsection{Ghost number twisting and cohomology}

The gauge fixed action can be expressed in terms of an unconstrained spacetime spinor $\theta^{\alpha}$, making spacetime supersymmetry manifest.

To see this, consider the field redefinitions

$$
\begin{aligned}
\lambda^{\alpha} & \rightarrow \gamma^{-1} \lambda^{\alpha}, \\
w_{\alpha} & \rightarrow \gamma w_{\alpha}, \\
\hat{\theta}^{\alpha} & \rightarrow \gamma \hat{\theta}^{\alpha}, \\
\hat{p}_{\alpha} & \rightarrow \gamma^{-1} \hat{p}_{\alpha} \\
\beta & \rightarrow \beta+\gamma^{-1} \lambda^{\alpha} w_{\alpha}+\gamma^{-1} \hat{\theta}^{\alpha} \hat{p}_{\alpha} .
\end{aligned}
$$

Although the action (3.28) is left unchanged, all spinors are now invariant under the scale transformations $(\Omega)$ with a consequent shift of their ghost number: $\lambda^{\alpha}\left(w_{\alpha}\right)$ has now ghost number $1(-1)$, while $\hat{\theta}^{\alpha}, \hat{p}_{\alpha}, \bar{\theta}^{\alpha}$, and $\bar{p}_{\alpha}$ have ghost number 0 .

The spacetime spinors $\left\{\hat{\theta}^{\alpha}, \hat{p}_{\alpha}, \bar{\theta}^{\alpha}, \bar{p}_{\alpha}\right\}$ can then combine into an unconstrained conjugate pair $\left\{\theta^{\alpha}, p_{\alpha}\right\}$, with the emergence of spacetime supersymmetry. The unconstrained spinors are defined as

$$
\begin{aligned}
\theta^{\alpha} & \equiv \hat{\theta}^{\alpha}+\bar{\theta}^{\alpha}, \\
p_{\alpha} & \equiv \hat{p}_{\alpha}+\bar{p}_{\alpha} .
\end{aligned}
$$

To show that $\left\{\theta^{\alpha}, p_{\alpha}\right\}$ are indeed unconstrained, first recall that, by construction, $\bar{p}_{\alpha}$ has a gauge invariance of the form $\delta \bar{p}_{\alpha}=e_{m}\left(\gamma^{m} \lambda\right)_{\alpha}$ due to the constraint $\left(\lambda \gamma^{m} \bar{\theta}\right)=0$. This leaves $\bar{\theta}^{\alpha}$ and $\bar{p}_{\alpha}$ with 11 independent components each. Due to the constraint $\left(\lambda \gamma^{m n} \hat{p}\right)=0$, $\hat{p}_{\alpha}$ has only 5 independent components, while the number of irreducible components in the twistor-like constraint (and consequently the number of independent components of the associated ghost, $\left.\hat{\theta}^{\alpha}\right)$ is also $5, c f$. section 2 . Their complementarity $(11+5=16)$ can be directly shown by the $\mathrm{SO}(10)$ spinor decomposition.

With the redefinition (3.34), note also that the ghost variables $\{\gamma, \beta, \Omega, \bar{\Omega}\}$ constitute a decoupled $\mathrm{U}(1)$ sector. After the addition of the null terms $\lambda^{\alpha} \hat{p}_{\alpha}$ and $\left(\lambda \gamma^{m} \bar{\theta}\right) P_{m}$, the BRST charge can be rewritten as

$$
Q=Q_{*}+Q_{P S}
$$

where

$$
\begin{aligned}
Q_{*} & =\Omega \beta \gamma, \\
Q_{P S} & =\lambda^{\alpha} d_{\alpha} .
\end{aligned}
$$

$Q_{P S}$ is the pure spinor BRST charge,with

$$
d_{\alpha} \equiv p_{\alpha}-P_{m}\left(\gamma^{m} \theta\right)_{\alpha} .
$$

Since $Q_{P S}$ and $Q_{*}$ are independent of each other, it is more convenient to analyze their cohomology separately. There are two elements in the cohomology of $Q_{*}$, the identity 
operator, $\mathbb{1}$, and $\Omega$. Any other $Q_{*}$-closed operator can be shown to be $Q_{*}$-exact. In fact, even the operator $\Omega$ is singular in the sense that it can be written as the limit of a $Q_{*}$-exact operator:

$$
\lim _{\epsilon \rightarrow 0}\left\{Q_{*}, \frac{1}{\epsilon} \gamma^{\epsilon}\right\}=\Omega
$$

The cohomology of $Q_{P S}$ is well known [10] and will be just quickly reviewed here. According to the pure spinor ghost number, it is organized as

$$
\left\{\mathbb{1}, \lambda^{\alpha} A_{\alpha}, \lambda^{\alpha} \lambda^{\beta} A_{\alpha \beta},\left(\lambda \gamma^{m} \theta\right)\left(\lambda \gamma^{n} \theta\right)\left(\lambda \gamma^{p} \theta\right)\left(\theta \gamma_{m n p} \theta\right)\right\} .
$$

At ghost number zero it contains only the identity operator. At ghost number one, $A_{\alpha}$ denotes the super Maxwell superfield and BRST closedness implies the equation of motion

$$
D_{\alpha} \gamma_{m n p q r}^{\alpha \beta} A_{\beta}=0
$$

where $D_{\alpha}$ is the superderivative given by

$$
D_{\alpha} \equiv \partial_{\alpha}+\left(\gamma^{m} \theta\right)_{\alpha} \partial_{m} .
$$

BRST-exact states account for the gauge transformation $\delta A_{\alpha}=D_{\alpha} \Lambda$, where $\Lambda$ is a superfield parameter. At ghost number two, $A_{\alpha \beta}$ denotes the superfield containing the super Maxwell antifields, and at ghost number three the only cohomology element is the so-called pure spinor measure factor.

Finally, the cohomology of the BRST charge (3.36) is given by a direct product of the cohomology of its two pieces, consisting of a doubling of the pure spinor superparticle cohomology: one sector completely independent of the $\mathrm{U}(1)$ variables $\{\gamma, \beta, \Omega, \bar{\Omega}\}$ and one sector linear in $\Omega$, both with the same physical content of (3.40).

\section{Summary and final remarks}

The BRST quantization of the massless particle action subjected to the constraint

$$
\bar{p}^{a}+\gamma^{a b} p_{b}=0,
$$

was shown here to give rise to an interesting structure (subsection 2.1). In particular, its ghost number zero cohomology is described by the wave function

$$
F=F\left(\mathbb{X}^{a}, \gamma^{a b}\right)
$$

which was used in [8] as a generating function for massless solutions of field equations of spin $\frac{N}{2}, c f$. equation (1.11).

Equation (4.1) was shown in subsection 2.2 to be equivalent to the twistor-like constraint

$$
\left(\gamma^{m} \lambda\right)_{\alpha} P_{m}=0,
$$

suggested as the fundamental gauge structure behind the pure spinor superparticle [4, 9]. However, cf. subsection 2.3, it does not seem to be possible to obtain the superparticle solely from gauge fixing the action (2.19) proposed in [9]. 
With the introduction of a partial worldline supersymmetry connecting the spacetime spinors (section 3), the BV quantization of the new model leads to the gauge fixed action

$$
S_{P S}=\int d \tau\left\{P_{m} \dot{X}^{m}-\frac{1}{2} P_{m} P^{m}+p_{\alpha} \dot{\theta}^{\alpha}+w_{\alpha} \dot{\lambda}^{\alpha}+\bar{\Omega} \dot{\Omega}+\beta \dot{\gamma}\right\}
$$

expressed in terms of the unconstrained spinors $\theta^{\alpha}$ and $p_{\alpha}$. Its BRST charge is given by the usual pure spinor BRST charge plus a U(1) decoupled sector, $c f$. equation (3.36).

It is interesting to observe the connections between different (super)particle descriptions. With the addition of a partial worldline supersymmetry to Berkovits' twistor-like constraint, spacetime supersymmetry emerges, giving rise to the pure spinor superparticle in $D=10$, which has the same physical spectrum of the Brink-Schwarz superparticle [13]. In turn, as demonstrated in [14], the Brink-Schwarz superparticle and the spinning particle [15], with worldline supersymmetry, are classically equivalent.

There are several directions to be explored that could help to understand these connections in more detail. For example, the higher ghost number cohomology of (2.8) was not determined here but it could be interesting to explore its field content using the techniques of [8] with a generating function with an intrinsic gauge structure. Concerning the action (3.2), its covariant quantization in even dimensions might lead to (alternative) superparticle descriptions in $D<10$. Another interesting idea is to enhance it with full worldline supersymmetry and to analyze the possible connections between the pure spinor superparticle and an extended version of the spinning particle. This idea is supported by a recent work relating the Ramond-Neveu-Schwarz and pure spinor superstrings [16].

Probably the most interesting direction to be investigated is the extension of the results of section 3 to the worldsheet, trying to understand the gauge fixing leading to the pure spinor superstring [12]. The results of [9] are likely to be completed using the model presented here. Partial computations already reveal the same pattern, and the string version of the constraints (3.19) seem to lead to a consistent generalization of the gauge fixed action (3.28). As already pointed out in $[9,11]$, reparametrization symmetry is redundant with the implementation of the twistor-like constraint, but at the worldsheet level this is more subtle. It would be interesting to have a (worldsheet) covariant derivation of all these results. Finally, the idea introduced in [9] that the Green-Schwarz and the pure spinor formalisms are but different gauge fixings of the same master action is worth investigating, although this does not look so straightforward considering the constraints (3.19).

\section{Acknowledgments}

I would like to thank Thales Azevedo, Nathan Berkovits, Kara Farnsworth and Ondřej Hulík for their comments and discussions, and especially Andrei Mikhailov for the valuable suggestions. This research has been supported by the Grant Agency of the Czech Republic, under the grant P201/12/G028.

Open Access. This article is distributed under the terms of the Creative Commons Attribution License (CC-BY 4.0), which permits any use, distribution and reproduction in any medium, provided the original author(s) and source are credited. 


\section{References}

[1] D.P. Sorokin, Superbranes and superembeddings, Phys. Rept. 329 (2000) 1 [hep-th/9906142] [INSPIRE].

[2] R. Penrose, Twistor algebra, J. Math. Phys. 8 (1967) 345 [InSPIRE].

[3] I. Bars and M. Picón, Single twistor description of massless, massive, AdS and other interacting particles, Phys. Rev. D 73 (2006) 064002 [hep-th/0512091] [INSPIRE].

[4] N. Berkovits, Pure spinors, twistors and emergent supersymmetry, JHEP 12 (2012) 006 [arXiv: 1105.1147] [INSPIRE].

[5] N.A. Nekrasov, Lectures on curved beta-gamma systems, pure spinors and anomalies, hep-th/0511008 [INSPIRE].

[6] L.P. Hughston, The wave equation in even dimensions, in Further advances in twistor theory, vol. 1, Res. Notes Math. 231, Longman, U.K., (1990), pg. 26 [ISBN-10:0470216557].

[7] L.P. Hughston, A remarkable connection between the wave equation and pure spinors in higher dimensions, in Further advances in twistor theory, vol. 1, Res. Notes Math. 231, Longman, U.K., (1990), pg. 37 [ISBN-10:0470216557].

[8] N. Berkovits and S.A. Cherkis, Higher-dimensional twistor transforms using pure spinors, JHEP 12 (2004) 049 [hep-th/0409243] [INSPIRE].

[9] N. Berkovits, Origin of the pure spinor and Green-Schwarz formalisms, JHEP 07 (2015) 091 [arXiv: 1503.03080] [INSPIRE].

[10] N. Berkovits, Covariant quantization of the superparticle using pure spinors, JHEP 09 (2001) 016 [hep-th/0105050] [INSPIRE].

[11] N. Berkovits, Twistor origin of the superstring, JHEP 03 (2015) 122 [arXiv:1409.2510] [INSPIRE].

[12] N. Berkovits, Super Poincaré covariant quantization of the superstring, JHEP 04 (2000) 018 [hep-th/0001035] [INSPIRE].

[13] L. Brink and J.H. Schwarz, Quantum superspace, Phys. Lett. B 100 (1981) 310 [InSPIRE].

[14] D.P. Sorokin, V.I. Tkach, D.V. Volkov and A.A. Zheltukhin, From the superparticle Siegel symmetry to the spinning particle proper time supersymmetry, Phys. Lett. B 216 (1989) 302 [INSPIRE].

[15] L. Brink, S. Deser, B. Zumino, P. Di Vecchia and P.S. Howe, Local supersymmetry for spinning particles, Phys. Lett. B 64 (1976) 435 [Erratum ibid. B 68 (1977) 488] [INSPIRE].

[16] N. Berkovits, Untwisting the pure spinor formalism to the RNS and twistor string in a flat and $A d S_{5} \times S^{5}$ background, JHEP 06 (2016) 127 [arXiv:1604.04617] [INSPIRE]. 